Research Article

\title{
A Study of Nil Ideals and Kothe's Conjecture in Neutrosophic Rings
}

\author{
Mohammad Abobala (iD \\ Tishreen University, Faculty of Science, Department of Mathematics, Lattakia, Syria \\ Correspondence should be addressed to Mohammad Abobala; mohammadabobala777@gmail.com
}

Received 31 March 2021; Revised 19 May 2021; Accepted 31 May 2021; Published 8 June 2021

Academic Editor: Theodore E. Simos

Copyright (c) 2021 Mohammad Abobala. This is an open access article distributed under the Creative Commons Attribution License, which permits unrestricted use, distribution, and reproduction in any medium, provided the original work is properly cited.

The aim of this study is to determine the necessary and sufficient condition for any AH subset to be a full ideal in a neutrosophic ring $R(I)$ and to be a nil ideal too. Also, this work shows the equivalence between Kothe's conjecture in classical rings and corresponding neutrosophic rings, i.e., it proves that Kothe's conjecture is true in the neutrosophic ring $R(I)$ if and only if it is true in the corresponding classical ring $R$.

\section{Introduction}

It was Zadeh [1] who introduced the notion of fuzzy sets to knob the uncertainties. Since then, the fuzzy set has been pragmatic in many commands such as medical diagnosis [2], decision making [3], and pattern recognition [4]. Keeping in view the prominence of fuzzy sets, many generalizations of fuzzy sets have been familiarized like rough sets [5], soft sets [6], intuitionistic fuzzy set [7], linear Diophantine fuzzy sets [8], bipolar valued fuzzy sets [9], bipolar soft sets [10, 11], picture fuzzy set [12], and spherical and T-spherical fuzzy sets [13]. Although all these generalizations have their own benefits, the notion of the neutrosophic set [14] has expanded much more responsiveness from the researchers as compared to the others [15].

Neutrosophy is a new branch of philosophy founded by Smarandache [16] to deal with indeterminacy in real life and scientific actions. It became a useful tool in many areas such as topology [17, 18], statistics [19], probability [20], and algebra [21-23].

Introducing neutrosophic algebras began with Smarandache and Kandasamy [24], where they presented concepts such as neutrosophic groups and neutrosophic rings and loops by inserting the indeterminacy element $I$ into classical algebraic structures.

In [25-27], we find two generalizations of neutrosophic rings, refined and $n$-refined neutrosophic rings, respectively. Many interesting properties of these kinds were discussed such as $\mathrm{AH}$ ideals, equations [28, 29], $\mathrm{AH}$ homomorphisms [30], and idempotency [31].

Neutrosophic rings were applicable in other algebraic structures, where neutrosophic modules, matrices [32-35], and $\mathrm{AH}$ spaces [36] can be built over these rings in a similar way to classical cases.

In [37], Abobala has proved that every neutrosophic ring is a homomorphic image of the corresponding refined neutrosophic ring. A similar relationship was proven in [38] for $n$-refined neutrosophic rings.

Recently, the structure of maximal and minimal full $\mathrm{AH}$ ideals of $n$-refined neutrosophic rings was presented [39]. Since, every neutrosophic ring $R(I)$ can be understood as $R(I)=R+R I=\{(a+b I) ; a, b \in R\}$.

Then, each AH subset of $R(I)$ is defined to have the form $M=P+S I ; P$ and $S$ are the two subsets of $R$. We call $P$ the real part and $S$ the neutrosophic part of $M$.

An important question arises here. The question is "When $M$ is a neutrosophic ideal of $R(I)$ ?" In other words, what conditions on the real part $P$ and neutrosophic part $S$ make $M$ be an ideal?

Also, it is clear that if Kothe's conjecture about nil ideals is true in $R(I)$, then it is true in $R$. What about the inverse relationship? In other words, if Kothe's conjecture is true in $R$, then is it true for $R(I)$ ?

These important questions motivate us to do this study, where we try to give full description of their answers. 


\section{Preliminaries}

Definition 1. Let $(R,+, \times)$ be a ring; then, $R(I)=\{a+b I ; a, b \in R\}$ is called the neutrosophic ring, where $I$ is a neutrosophic element with the condition $I^{2}=I$.

If $R$ is a field, then $R(I)$ is called a neutrosophic field. A neutrosophic field is not a field by the classical meaning, since $I$ is not invertible.

Definition 2. Let $R$ be a ring and $R(I)$ be the related neutrosophic ring, and $P=P_{0}+P_{1} I=\left\{a_{0}+a_{1} I ; a_{0} \in P_{0}\right.$, $\left.a_{1} \in P_{1}\right\} ; P_{0}$, and $P_{1}$ are the two subsets of $R$; then, $P$ is called an $\mathrm{AH}$ subset.

(a) We say that $P$ is an AH ideal if $P_{0}$ and $P_{1}$ are ideals in the ring $R$

(b) We say that $P$ is an AHS ideal if $P_{0}=P_{1}$

Definition 3. Let $R(I)$ be any neutrosophic ring, and $P=$ $P_{0}+P_{1} I$ be any AH subset. We say that $P$ is a full ideal of $R(I)$ if it is an ideal by classical meaning.

Definition 4. The element $I$ can be split into two indeterminacies $I_{1}$ and $I_{2}$ with the following conditions:

$$
\begin{aligned}
I_{1}^{2} & =I_{1}, \\
I_{2}^{2} & =I_{2}, \\
I_{1} I_{2} & =I_{2} I_{1}=I_{1} .
\end{aligned}
$$

Definition 5. If $X$ is a set, then $X\left(X\left(I_{1}, I_{2}\right)=\right.$ $\left\{\left(a, b I_{1}, c I_{2}\right): a, b, c \in X\right\}$ is called the refined neutrosophic set generated by $X, I_{1}$, and $I_{2}$.

Definition 6. Let $(R,+, \times)$ be a ring, and $\left(R\left(I_{1}, I_{2}\right),+, \times\right)$ is called a refined neutrosophic ring generated by $R, I_{1}, I_{2}$.

Theorem 1. Let $\left(R\left(I_{1}, I_{2}\right),+, \times\right)$ be a refined neutrosophic ring; then, it is a ring. field.

It is called a refined neutrosophic field if $R$ is a classical

2.1. Kothe Conjecture. If $R$ is a ring, then the sum of any two left nil ideals is nil again. For more equivalent forms of Kothe conjecture, see [20].

\section{Main Discussion}

First of all, we determine the conditions of AH subsets to be ideals in the neutrosophic rings with unity.

Theorem 2. Let $R(I)$ be a neutrosophic ring with unity 1 and $M=P+S I$ be any $A H$ subset of $R(I)$; then, $M$ is a neutrosophic ideal (full ideal) if and only if the following conditions are true:

(a) $P$ is an ideal on $R$ (b) $P$ is contained in $S$

(c) $S$ is an ideal of $R$

Proof. First, we assume that (a), (b), and (c) are true; we have $(M,+)$ is a subgroup of $(R(I),+)$, that is, because if $a+b I, c+\mathrm{d} I \in M ; a, c \in P, b, d \in S$, we find

$$
(a+b I)-(c+d I)=(a-c)+(b-d) I \in M ; \quad a-c \in P, b-d \in S .
$$

Now, suppose that $a+b I \in M$ and $r=m+n I \in R(I)$, we have $r \cdot(a+b I)=m \cdot a+I[m \cdot b+n \cdot b+n \cdot a]$; by the assumption, we regard that $m \cdot b+n \cdot b \in S$ and $n \cdot a \in P \leq S$; thus, $r \cdot(a+b I)=m \cdot a+I[m \cdot b+n \cdot b+n \cdot a] \in P+S I=$ $M$, which means that $M$ is a neutrosophic ideal of $R(I)$.

Conversely, we suppose that $M=P+S I$ is a neutrosophic ideal of $R(I)$. Let $a$ and $c$ be two arbitrary elements in $P$, and $b$ and $d$ be two arbitrary elements in $S$; we have $a+b I, c+d I \in M$; by using the assumption, we have $M$ as an ideal; hence, $(a+b I)-(c+d I)=(a-c)+(b-d)$ $I \in M=P+S I$; thus,

$$
\begin{aligned}
& a-c \in P, \\
& b-d \in S,
\end{aligned}
$$

and $(P,+)$ and $(S,+)$ are the two subgroups of $(R,+)$.

For every $r \in R$, we have $r=r+0 I \in R(I)$ and $r$. $(a+b I)=r \cdot a+r \cdot b I \in M=P+S I$; thus, $r \cdot a \in P$ and $r$. $b \in S$; this means that $P$ and $S$ are the ideals in the classical ring $R$.

Now, we prove that $P$ is contained in $S$. We have $(1-I) \in R(I)$, that is, because $R(I)$ has a unity 1 . On the other hand, we can write $(1-I)(a+b I)=(a-a I)$ $\in M=P+S I$, that is, because $M$ is an ideal of $R(I)$; hence, $-a \in S$, and thus, $a \in S$; by regarding that $a$ is an arbitrary element of $P$, we get that $P \leq S$.

Example 1. Let $R=Z$ be the ring of integers, and $R(I)=$ $Z(I)=\{a+b I ; a, b \in Z\}$ be the corresponding neutrosophic ring, we have

(a) $P=\langle 2\rangle, Q=\langle 4\rangle$, and $S=\langle 3\rangle$ are the three ideals of $R$, with $Q \leq P$

(b) $M=Q+P I=\{4 m+2 n I ; m, n \in Z\}$ is an ideal of $R(I)$

(c) $N=P+S I=\{2 m+3 n I ; m, n \in Z\}$ is not a neutrosophic ideal, that is, because $P$ is not contained in $S$

Example 2. Let $R=Z_{8}$ be the ring of integers modulo 8, and $R(I)=\left\{a+b I ; a, b \in Z_{8}\right\} \quad$ be the corresponding neutrosophic ring. Consider the set $M=\{0,4,2 I, 4 I, 6 I$, $4+2 I, 4+6 I, 4+4 I\}$. We have $M$ as an ideal of $R(I)$, that is, because $M=\langle 4\rangle+\langle 2\rangle I$ and $\langle 4\rangle \leq\langle 2\rangle$.

Now, we show that Theorem 2 is not true in the case of neutrosophic rings with no unity by the following counter example. 
Example 3. Consider the ring $R=2 Z=\{2 m ; m \in Z\}$, and it is clear that it has no unity.

Let $Q_{0}=R$ and $Q_{1}=\{4 n ; n \in Z\}$ be an ideal in $R$. It is clear that $Q_{0}$ is not contained in $Q_{1}$. Now, we should check the set $Q=Q_{0}+Q_{1} I$ if it makes an ideal of $R(I)$ or not.

First of all, it is easy to see that $Q$ is a subgroup with respect to addition of the additive group $(R(I),+)$.

On the other hand, we assume that $x=2 n+4 m I \in Q$ and $r=2 d+2 c I$ is an arbitrary element of $R(I)=2 Z(I)$. We have

$$
\begin{aligned}
r \cdot x= & 4 n d+I(8 m d+8 m c+4 n c)=2(2 n d) \\
& +4[2 m d+2 m c+n c] I \in Q .
\end{aligned}
$$

Hence, $Q$ is an ideal of $R(I)$.

Now, we show that the necessary and sufficient conditions on any AH subset $P=P_{0}+P_{1} I$ to be an ideal in $R(I)$ if $R(I)$ has no unity.

Theorem 3. Let $R$ be any ring without unity and $R(I)$ be its corresponding neutrosophic ring. Then, the AH subset $P=$ $P_{0}+P_{1} I$ is an ideal of $R(I)$ if and only if

(a) $P_{0}$ and $P_{1}$ are ideals of $R$

(b) For every $x \in P_{0}$ and $r \in R$, we have $r x \in P_{1}$

Proof. We suppose that $P$ is a full ideal of $R(I)$; hence, $P_{0}$ and $P_{1}$ are the ideals of $R$ by a similar proof of Theorem 2 . On the other hand, for every $x_{0}+y_{0} I \in P$ and $r+$ $r I \in R(I)$, where $r \in R$, we have $(r+r I)\left(x_{0}+y_{0} I\right) \in P$; thus $r x_{0}+I\left(r y_{0}+r y_{0}+r x_{0}\right) \in P$. This means that $r x_{0} \in P_{1}$, that is, because $y_{0} \in P_{1}$.

For the converse, we assume that conditions (a) and (b) are true; hence, it is clear that $(P,+)$ is a subgroup of $(R(I),+)$. Now, consider an arbitrary element $x_{0}+y_{0} I \in P$ and any $r=m+n I \in R(I)$; we have $r\left(x_{0}+y_{0} I\right)=(m+n I)$ $\left(x_{0}+y_{0} I\right)=m x_{0}+I\left(m y_{0}+n x_{0}+n y_{0}\right)$; by the assumption that (a) and (b) are true, we get $n x_{0} \in P_{1}$; hence, $m x_{0}+I\left(m y_{0}+n x_{0}+n y_{0}\right) \in P$, and $P$ is an ideal of $R(I)$.

The following theorem describes the structure of nil $\mathrm{AH}$ ideals in $R(I)$.

Theorem 4. Let $R(I)$ be any neutrosophic ring, we have

(a) $x+y I$ is nilpotent in $R(I)$ if and only if $x, x+y$ are nilpotent elements in $R$

(b) If $P=P_{0}+P_{1} I$ is a full ideal of $R(I)$, then $P$ is nilpotent if and only if $P_{0}, P_{1}$, and $P_{0}+P_{1}$ are nilpotent

(c) If $P=P_{0}+P_{1} I$ is a right/left full ideal of $R(I)$, then $P$ is nil if and only if $P_{0}, P_{1}, P_{0}+P_{1}$ are nil

\section{Proof}

(a) First of all, we prove that $(x+y I)^{n}=x^{n}+$ $\left[(x+y)^{n}-x^{n}\right] I$, where $n$ is any positive integer

For $n=1$, it is clear. We suppose that it is true for $n=k$, we shall prove it for $k+1$.

$$
\begin{aligned}
(x+y I)^{k+1}= & (x+y I)^{k}(x+y I) \\
= & \left(x^{k}+\left[(x+y)^{k}-x^{k}\right] I\right) \cdot(x+y I) \\
= & x^{k+1}+I\left[x^{k} \cdot y+(x+y)^{k} \cdot y+(x+y)^{k}\right. \\
& \left.\cdot x-x^{k} \cdot x-x^{k} \cdot y\right] \\
= & x^{k+1}+I\left[(x+y)^{k+1}-x^{k+1}\right] .
\end{aligned}
$$

Thus, it is true by induction

Now, we suppose that $x+y I$ is nilpotent in $R(I)$; hence, there is a positive integer $n$, such that $(x+y I)^{n}=0$. By the previous statement, we get $x^{n}=0$ and $(x+y)^{n}-x^{n}=0$; thus, $(x+y)^{n}=0$.

Thus, $x$ and $x+y$ are the nilpotent elements in $R$. The converse is clear.

(b) Let $P=P_{0}+P_{1} I$ be a nilpotent ideal of $R(I)$; then, there is a positive integer $n$, such that $P^{n}=\{0\}$. For any element $x \in P_{0}$, we have $x \in P$; hence, $x^{n}=\{0\}$. On the other hand, for any element $y \in P_{1}$, we have $y I \in P_{1} I \leq P$; hence, $(y I)^{n}=y^{n} I=\{0\}$, and $y^{n}=0$. This means that $P_{0}$ and $P_{1}$ are nilpotent. Also, we have that $(x+y)^{n}=0$, as a direct result of the equation $(x+y I)^{n}=x^{n}+\left[(x+y)^{n}-x^{n}\right] I$; hence, $P_{0}+P_{1}$ is nilpotent.

For the converse, we assume that $P_{0}, P_{1}$, and $P_{0}+$ $P_{1}$ are nilpotent ideals of $R$; then, there are two positive integers $m$ and $n$, such that $P_{0}^{n}=\left(P_{0}+\right.$ $\left.P_{1}\right)^{m}=\{0\}$. Hence, for every element $x+y I \in P$, we have $(x+y I)^{n+m}=x^{n+m}+\left[(x+y)^{n+m}-x^{n+m}\right]$ $I=0$. This implies that $P^{n+m}=\{0\}$, that is, because $x^{n+m}=(x+y)^{n+m}=0$, and $P$ must be a nilpotent ideal.

(c) First of all, we assume that $P=P_{0}+P_{1} I$ is nil in $R(I)$; hence, $P_{0}$ and $P_{1} I$ are nil as a direct result from the inclusion $P_{0} \leq P$ and $P_{1} I \leq P$; thus, $P_{0}$ and $P_{1}$ are nil in $R$. To prove that the sum $P_{0}+P_{1}$ is nil, we consider two arbitrary elements $x \in P_{0}$ and $y \in P_{1}$; hence, $x+y I \in P$, but $P$ is nil; thus, there is a positive integer $n$, such that $(x+y I)^{n}=0$; this implies that $x^{n}=0$ and $(x+y)^{n}-x^{n}=0$; thus, $(x+y)^{n}=0$, so we get $P_{0}+P_{1}$ is nil. For the converse, we suppose that $P_{0}, P_{1}$, and $P_{0}+P_{1}$ are nil ideals in $R$, and we shall prove that $P$ is nil.

Let $x+y I$ be an arbitrary element of $P$; we have $x \in P_{0}$ and $y \in P_{1}$. Under the assumption of theorem, we can find two positive integers $m$ and $n$, such that $x^{n}=(x+y)^{m}=0$. According to (a), we have $(x+y I)^{n+}$ $m=x^{n+m}+\left[(x+y)^{n+m}-x^{n+m}\right] I=(x+y)^{n+m} I=(x+y)^{n}$ $(x+y)^{m} I=0$. Thus, $P$ is a nil ideal of $R(I)$.

The following theorem shows the equivalence between Kothe's conjecture in the classical ring $R$ and the corresponding neutrosophic ring $R(I)$.

Theorem 5. Kothe's conjecture is true in $R(I)$ if and only if it is true in $R$ 
Proof. According to Theorem 4, we have $P=P_{0}+P_{1} I$ is nil in $R(I)$ if and only if $P_{0}, P_{1}$, and $P_{0}+P_{1}$ are nil in $R$. Thus, the sum of two left nil ideals $P=P_{0}+P_{1} I$ and $Q=Q_{0}+Q_{1} I$, say it is $S=\left(P_{0}+Q_{0}\right)+\left(P_{1}+Q_{1}\right) I$ in $R(I)$ is nil if and only if the following three sums $P_{0}+Q_{0}, P_{1}+Q_{1}$, and $P_{0}+Q_{0}+$ $P_{1}+Q_{1}$ are nil in $R$; hence, Kothe's conjecture is true in $R(I)$ if and only if it is true in $R$.

Theorem 6. If Kothe's conjecture is true in the refined neutrosophic ring $R\left(I_{1}, I_{2}\right)$ or in the n-refined neutrosophic ring $R_{n}(I)$, then it is true in the ring $R$.

Proof. According to $[37,38]$, every neutrosophic $\operatorname{ring} R$ is a homomorphic image of its corresponding refined neutrosophic ring $R\left(I_{1}, I_{2}\right)$ and $n$-refined neutrosophic ring $R_{n}(I)$; thus, if Kothe's conjecture holds in $R\left(I_{1}, I_{2}\right), R_{n}(I)$, then it holds in $R$.

Remark 1. It is still unknown that if Kothe's conjecture is true in $R$, then it is true in $R\left(I_{1}, I_{2}\right), R_{n}(I)$.

3.1. Open Questions. Two interesting open questions are coming to light according to this work. The first can be asked as follows. If Kothe's conjecture is true in the $\operatorname{ring} R$, then is it true in the corresponding refined neutrosophic ring $R\left(I_{1}, I_{2}\right)$. The second is the following. If Kothe's conjecture is true in $R$, then is it true in the corresponding $n$-refined neutrosophic ring $R_{n}(I)$. These open questions maybe the future of the study of Kothe conjecture in neutrosophic ring theory.

\section{Data Availability}

The data used to support the findings of this study are available from the corresponding author upon request.

\section{Conflicts of Interest}

The author declares that there are no conflicts of interest.

\section{References}

[1] L. A. Zadeh, "Fuzzy sets," Information and Control, vol. 8, no. 3, pp. 338-353, 1965.

[2] J. F.-F. Yao and J.-S. Yao, "Fuzzy decision making for medical diagnosis based on fuzzy number and compositional rule of inference," Fuzzy Sets and Systems, vol. 120, no. 2, pp. 351-366, 2001.

[3] P. Y. Ekel, "Fuzzy sets and models of decision making," Computers \& Mathematics with Applications, vol. 44, no. 7, pp. 863-875, 2002.

[4] W. Pedrycz, "Fuzzy sets in pattern recognition: methodology and methods," Pattern Recognition, vol. 23, no. 1-2, pp. 121-146, 1990.

[5] Z. a. Pawlak, "Rough sets," International Journal of Computer \& Information Sciences, vol. 11, no. 5, pp. 341-356, 1982.

[6] D. Molodtsov, "Soft set theory-first results," Computer and Mathematics with Applications, vol. 37, no. 4-5, pp. 19-31, 1999.
[7] K. T. Atanassov, "Intuitionistic fuzzy sets," Fuzzy Sets and Systems, vol. 20, no. 1, pp. 87-96, 1986.

[8] M. Riaz and M. R. Hashmi, "Linear diophantine fuzzy set and its applications towards multi-attribute decision-making problems," Journal of Intelligent and Fuzzy Systems, vol. 37, no. 4, pp. 5417-5439, 2020.

[9] K. M. Lee, "bulletin," Fuzzy Sets and Systems, vol. 110, no. 2, pp. 307-312, 2000.

[10] M. Shabir and M. Naz, "On bipolar soft sets," 2013, https:// arxiv.org/abs/1303.1344.

[11] T. Mahmood, "A novel approach towards bipolar soft sets and their applications," Journal of Mathematics, vol. 2021, Article ID 4690808, 11 pages, 2020.

[12] B. C. Cuong, Picture Fuzzy Sets-First Results. Pa 1, Inpreprint of Seminar on Neuro-Fuzzy Systems with Applications, Institute of Mathematics, Hanoi, Vietnam, 2013.

[13] T. Mahmood, T. Ullah, Q. Khan, and N. Jan, "An approach towards decision making and medical diagnosis problems using the concept of spherical fuzzy sets," Neural Computing and Applications, vol. 31, pp. 7041-7053, 2019.

[14] F. Smarandache, Neutrosophy Neutrosophic Probability, Set and Logic: Analytic Synthesis Synthetic Analysis, American Research Press, Santa Fe, NM, USA, 1998.

[15] F. Smarandache, "Neutrosophic set a generalization of the intuitionistic fuzzy sets," Indian Journal of Pure and Applied Mathematics, vol. 20, pp. 287-297, 2005.

[16] F. Smarandache, "n-Valued refined neutrosophic logic and its applications in physics," Progress in Physics, vol. 4, pp. 143146, 2013.

[17] M. . Akram, H. . Gulzar, and F. Smarandache, "Neutrosophic soft topological K-algebras," Neutrosophic Sets and Systems, vol. 25, pp. 104-124, 2020.

[18] K. Ramesh, "Ngbr homomorphisms in neutrosophic topological spaces," Neutrosophic Sets and Systems, vol. 32, pp. 25-37, 2020.

[19] R. Alhabib and A. A. Salama, "The neutrosophic time seriesstudy its models (linear-logarithmic) and test the coefficients significance of its linear model," Neutrosophic Sets and Systems, vol. 33, pp. 105-115, 2020.

[20] A. Smoktunowicz, "On some results related to kothe's conjecture," Serdica Mathematical Journal, vol. 27, pp. 159-170, 2001.

[21] T. Chalapathi and L. Madhavi, "Neutrosophic boolean rings," Neutrosophic Sets and Systems, vol. 33, pp. 57-66, 2020.

[22] S. A. Edalatpanah, "Systems of neutrosophic linear equations," Neutrosophic Sets and Systems, vol. 33, pp. 92-104, 2020.

[23] M. A. Ibrahim, A. A. A. Agboola, B. S. Badmus, and S. A. Akinleye, "On refined neutrosophic vector spaces II," International Journal of Neutrosophic Science, vol. 9, pp. 2236, 2020.

[24] W. B. V. Kandasamy and F. Smarandache, Neutrosophic Rings, Infinite Study, Hexis, Phoenix, Arizona, 2006.

[25] E. O. Adeleke, A. A. A. Agboola, and F. Smarandache, "Refined neutrosophic rings I," International Journal of Neutrosophic Science, vol. 2, pp. 77-81, 2020.

[26] F. Smarandache and M. Abobala, " $n$-refined neutrosophic rings," International Journal of Neutrosophic Science, vol. 6, pp. 83-90, 2020.

[27] E. O. Adeleke, A. A. A. Agboola, and F. Smarandache, "Refined neutrosophic rings II," International Journal of Neutrosophic Science, vol. 2, pp. 89-94, 2020.

[28] M. Abobala, "On some algebraic properties of n-refined neutrosophic elements and $n$-refined neutrosophic linear 
equations," Mathematical Problems in Engineering, Article ID 5573072, 7 pages, 2021.

[29] H. Sankari and M. Abobala, "Neutrosophic linear diophantine equations with two variables," Neutrosophic Sets and Systems, vol. 38, pp. 1-401, 2020.

[30] H. Sankari and M. Abobala, "AH-homomorphisms in neutrosophic rings and refined neutrosophic rings," Neutrosophic Sets and Systems, vol. 38, pp. 1-526, 2020.

[31] V. W. B. Kandasamy, K. Ilanthenral, and F. Smarandache, "Semi-idempotents in neutrosophic rings," Mathematics Journal (MDPI), vol. 7, no. 6, p. 506, 2019.

[32] M. Abobala, "On refined neutrosophic matrices and their applications in refined neutrosophic algebraic equations," Journal of Mathematics, Article ID 5531093, 5 pages, 2021.

[33] M. Abobala, A. Hatip, N. Olgun, S. Broumi, A. ., A. Salama, and E. H. Khaled, "The algebraic creativity in the neutrosophic square matrices," Neutrosophic Sets and Systems, vol. 40, pp. 1-11, 2021.

[34] A. Hatip and N. Olgun, "On refined neutrosophic R-module," International Journal of Neutrosophic Science, vol. 7, pp. 8796, 2020.

[35] R. Das, F. Smarandache, and B. Tripathy, "Neutrosophic fuzzy matrices and some algebraic operations," Neutrosophic Sets and Systems, vol. 32, pp. 401-409, 2020.

[36] M. Abobala, "On the representation of neutrosophic matrices by neutrosophic linear transformations," Journal of Mathematics, vol. 2021, Article ID 5591576, 5 pages, 2021.

[37] M. Abobala, "Classical homomorphisms between refined neutrosophic rings and neutrosophic rings," International Journal of Neutrosophic Science, vol. 5, pp. 72-75, 2020.

[38] M. Abobala, "Classical homomorphisms between n-refined neutrosophic rings," International Journal of Neutrosophic Science, vol. 7, pp. 74-78, 2020.

[39] M. Abobala, "A study of maximal and minimal ideals of n-refined neutrosophic rings," Journal of Fuzzy Extension and Applications, vol. 2, pp. 16-22, 2021. 